\title{
Congenital primary megaureter
}

INSERM

\section{Source}

INSERM. (1999). Orphanet: an online rare disease and orphan drug data base. Congenital primary megaureter. ORPHA:617

Congenital primary megaureter (PM) is an idiopathic condition in which the bladder and bladder outlet are normal but the ureter is dilated to some extent. It may be obstructed, refluxing or unobstructed and not refluxing. 